\title{
ORTHOGONALLY ADDITIVE HOLOMORPHIC FUNCTIONS OF BOUNDED TYPE OVER $C(K)$
}

\author{
DANIEL CARANDO, SILVIA LASSALLE, AND IGNACIO ZALDUENDO
}

\begin{abstract}
It is known that all $k$-homogeneous orthogonally additive polynomials $P$ over $C(K)$ are of the form

$$
P(x)=\int_{K} x^{k} d \mu .
$$

Thus $x \mapsto x^{k}$ factors all orthogonally additive polynomials through some linear form $\mu$. We show that no such linearization is possible without homogeneity. However, we also show that every orthogonally additive holomorphic functions of bounded type $f$ over $C(K)$ is of the form

$$
f(x)=\int_{K} h(x) d \mu
$$
\end{abstract}

for some $\mu$ and holomorphic $h: C(K) \rightarrow L^{1}(\mu)$ of bounded type.

\section{INTRODUCTION.}

A holomorphic function $f: C(K) \rightarrow F$ is said to be orthogonally additive if $f(x+y)=f(x)+f(y)$ whenever $x$ and $y$ are mutually orthognal (i.e., $x y=0$ over $K$ ). In this paper we study orthogonal additivity of complex-valued bounded-type holomorphic functions over $C(K)$.

Recall [8] that $f: E \rightarrow F$ is of bounded type if it is bounded on all bounded subsets of $E$. The Taylor series $f=\sum_{k} P_{k}$ of such a function have infinite radii of uniform convergence, i.e.: $\lim \sup \left\|P_{k}\right\|^{\frac{1}{k}}=0$. We will denote the space of all such functions over $E$ by $\mathcal{H}_{b}(E, F)$, or simply $\mathcal{H}_{b}(E)$ if $F=\mathbb{C}$.

It was proved by [9] and [2] (see also [3]) that each orthogonally additive $k$-homogeneous polynomial $P$ over $C(K)$ is represented by a

Key words and phrases. orthogonally additive, holomorphic functions over $C(K)$.

Partially supported by PICT 05 33042. The first author was supported in part by UBACyT X038 and PICT 06-00587. The second author was also supported by UBACyT X863 and PICT 06-00897. The three authors are members of CONICET. 
regular Borel measure $\mu$ on $K$, in the following sense:

$$
P(x)=\int_{K} x^{k} d \mu \quad \text { for all } x \in C(K) .
$$

This is a linearization result; the 'universal' polynomial $h(x)=x^{k}$ linearizes all orthogonally additive $k$-homogeneous polynomials $P$,

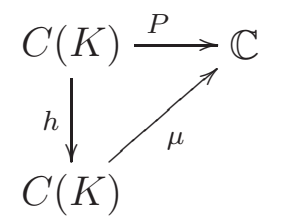

The following lemma shows that $f$ is orthogonally additive if and only if all polynomials in its Taylor series expansion at zero are orthogonally additive. Note that this is not the case for expansions around other points.

Lemma 0.1. Let $f: C(K) \rightarrow \mathbb{C}$ be holomorphic, and $f=\sum_{k=0}^{\infty} P_{k}$ its Taylor series at zero. Then $f$ is orthogonally additive if and only if all the $P_{k}$ 's are orthogonally additive.

Proof. Say $x$ and $y$ are orthogonal. If $f$ is orthogonally additive, we may write

$$
\begin{aligned}
P_{k}(x+y) & =\frac{1}{k !} \frac{d^{k}}{d \lambda}(0)(f(\lambda(x+y))) \\
& =\frac{1}{k !} \frac{d^{k}}{d \lambda}(0)(f(\lambda x)+f(\lambda y)) \\
& =\frac{1}{k !} \frac{d^{k}}{d \lambda}(0) f(\lambda x)+\frac{1}{k !} \frac{d^{k}}{d \lambda}(0) f(\lambda y) \\
& =P_{k}(x)+P_{k}(y) .
\end{aligned}
$$

For the converse, if all $P_{k}$ 's are orthogonally additive,

$$
\begin{aligned}
f(x+y) & =\sum_{k=0}^{\infty} P_{k}(x+y)=\sum_{k=0}^{\infty}\left(P_{k}(x)+P_{k}(y)\right) \\
& =\sum_{k=0}^{\infty} P_{k}(x)+\sum_{k=0}^{\infty} P_{k}(y) \\
& =f(x)+f(y) .
\end{aligned}
$$

Given the Lemma, one might expect that in the holomorphic setting one will have a linearizing factorization where the polynomial $h(x)=$ $x^{k}$ is replaced by $h(x)=\Phi \circ x$, with $\Phi: \mathbb{C} \rightarrow \mathbb{C}$ holomorphic and 
$\Phi(0)=0$ (for example $\left.h(x)=e^{x}-1\right)$. The first objection to such a factorization is that it would produce a linearization of the space of orthogonally additive holomorphic functions through $C(K)$, and thus a Banach predual of the space; but spaces of holomorphic functions are seldom Banach. A second objection to this line of thought is that for any fixed $\Phi(z)=\sum_{k=1}^{\infty} a_{k} z^{k}$, the factorization can only take place for functions $f$ with limited growth: indeed, if

$$
f(x)=\int_{K} \Phi \circ x d \mu=\int_{K} \sum_{k=1}^{\infty} a_{k} x^{k} d \mu=\sum_{k=1}^{\infty} a_{k} \int_{K} x^{k} d \mu,
$$

then

$$
|f(x)| \leq \sum_{k=1}^{\infty}\left|a_{k}\right||\mu|(K)\|x\|^{k}=|\mu|(K) \sum_{k=1}^{\infty}\left|a_{k}\right|\|x\|^{k},
$$

so the growth of $f$ is bounded by that of $\Phi$.

We will show (Example 1) that there are stronger, algebraic, obstructions and that such a factorization cannot be obtained even for non-homogeneous polynomials of degree two.

In Section 1 we study the relationship between orthogonal additivity and integrality, and prove that all orthogonally additive functions of bounded type are integral. We also show an orthogonally additive polynomial of degree two that does not factor through $C(K)$.

In Section 2 we characterize orthogonally additive holomorphic functions of bounded type as those which may be written as

$$
f(x)=\int_{K} h(x) d \mu \quad \text { for all } x \text { in } C(K),
$$

for some measure $\mu$ on $K$ and $h: C(K) \rightarrow L^{1}(\mu)$, holomorphic and of bounded type.

\section{Orthogonal ADDitivity AND INTEGRALITY}

Recall [6] that a $k$-homogeneous polynomial $P$ over a Banach space $E$ is said to be integral if it can be represented by a Borel measure $\nu$ over the unit ball $B_{E^{\prime}}$ of the dual space (with the weak* topology) in the following way

$$
P(x)=\int_{B_{E^{\prime}}} \widehat{x}^{k} d \nu \quad \text { for all } x \in E
$$

(where $\widehat{x}(\gamma)=\gamma(x)$ ). Recall also [5] that an integral holomorphic function $f: B_{E}^{\circ} \rightarrow \mathbb{C}$ on the open unit ball of $E$ is one which may be written as

$$
f(x)=\int_{B_{E^{\prime}}} \frac{1}{1-\widehat{x}} d \nu \quad \text { for all } x \in B_{E}^{\circ} .
$$


and some Borel measure $\nu$ on $B_{E^{\prime}}$.

Note that when $E=C(K)$, the dual $E^{\prime}$ is the space of regular Borel measures on $K$, and that $K$ may be identified with part of the unit ball $B_{E^{\prime}}$ : the point measures $\delta_{a}, a \in K$.

We begin with some observations on the case of orthogonally additive $k$-homogeneous polynomials. Consider the closed subspace $X_{k}$ of $C\left(B_{E^{\prime}}\right)$ spanned by $\left\{\widehat{x}^{k}: x \in E\right\}$. The space $X_{k}$ is then the symmetric $\epsilon$-tensor product $\widehat{\bigotimes}_{\epsilon, k, s} E$ and integral $k$-homogeneous polynomials are those which linearize through $X_{k}$ [8].

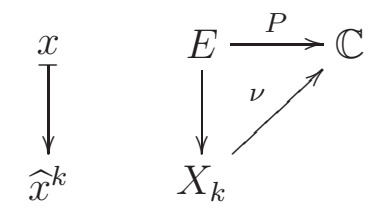

Now if $E=C(K)$, we have the linear map $R_{k}: X_{k} \rightarrow C(K)$ given by $R_{k}\left(\widehat{x}^{k}\right)=x^{k}$. It is easy to see that, since $K \subset B_{E^{\prime}}$, this map is well-defined and has norm one. The diagram

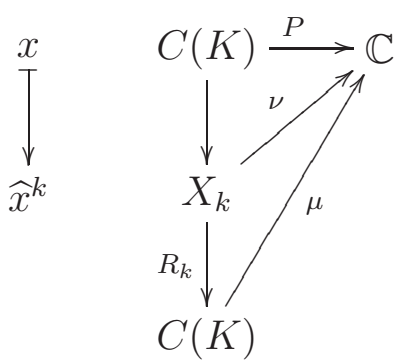

then shows (applying [9], [2]) that all orthogonally additive $k$-homogeneous polynomials are integral. Indeed, if $P(x)=\mu\left(x^{k}\right)$, it is also $\nu\left(\widehat{x}^{k}\right)$. This shows the well known fact that the 'integrating' polynomial $P(x)=$ $\int_{K} x(t)^{k} d \mu(t)$ on $C(K)$ is integral.

It is also true that orthogonally additive holomorphic functions of bounded type are integral. For the proof, we will need the following. Recall [1] that any $f \in \mathcal{H}_{b}(E)$ has a canonical extension to the bidual $E^{\prime \prime}$. This extension, $\bar{f}$, called the Aron-Berner extension is also holomorphic and of bounded type.

Given a Borel subset $A$ of $K$, and $f: C(K) \rightarrow \mathbb{C}$ a bounded-type holomorphic function, we define

$$
f_{A}(x)=\bar{f}\left(1_{A} x\right)
$$

where $\bar{f}$ is the Aron-Berner extension of $f$.

Proposition 1.1. If $f \in \mathcal{H}_{b}(C(K))$ is orthogonally additive, then it is integral. 
Proof. Let $f=\sum_{k=1}^{\infty} P_{k}$ be the Taylor series expansion of $f$ at zero, and note that each $P_{k}$ is orthogonally additive and hence integral. By [5] we need only verify that $\sum_{k=1}^{\infty}\left\|P_{k}\right\|_{I}<\infty$. Since $f$ is of bounded type, we have $\limsup \left\|P_{k}\right\|^{\frac{1}{k}}=0$, so $\sum_{k=1}^{\infty}\left\|P_{k}\right\|<\infty$. But if $P$ is an orthogonally additive $k$-homogeneous polynomial, then its natural and integral norms are equal: In general, one has $\|P\| \leq\|P\|_{I}$; to see $\|P\|_{I} \leq\|P\|$, given $\varepsilon>0$, we find a measure $\nu$ on $B_{E^{\prime}}$ representing $P$ and such that $|\nu|<\|P\|+\varepsilon$ : since $P$ is orthogonally additive, consider a measure $\mu$ on $K$ such that $P(x)=\int_{K} x^{k} d \mu$, and take $\nu=\mu \circ R_{k}$ :

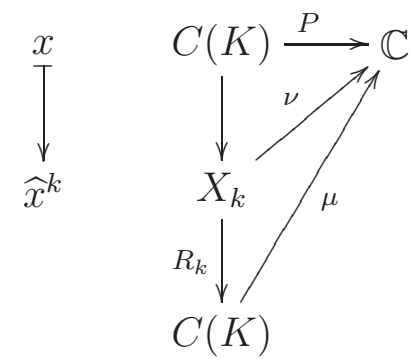

Since $\left\|R_{k}\right\|=1,|\nu|=\left\|\mu \circ R_{k}\right\| \leq|\mu|\left\|R_{k}\right\|=|\mu|<\sum_{i=1}^{n}\left|\mu\left(A_{i}\right)\right|+\varepsilon$ for some sequence $\left(A_{i}\right)$ of disjoint closed subsets of $K$. Hence,

$$
\begin{aligned}
|\nu| & <\sum_{i=1}^{n}\left|\int_{K} 1_{A_{i}} d \mu\right|+\varepsilon=\sum_{i=1}^{n}\left|P_{A_{i}}(1)\right|+\varepsilon \\
& \leq \sum_{i=1}^{n}\left\|P_{A_{i}}\right\|+\varepsilon \\
& \leq\|P\|+\varepsilon,
\end{aligned}
$$

this last inequality by Lemma 1.2 of [3].

Note that in the proof we have shown that the natural and integral norms coincide on the space of orthogonally additive $k$-homogenous polynomials on $C(K)$. We have also shown that any orthogonally additive function in $\mathcal{H}_{b}(C(K))$ is, in fact, in $\mathcal{H}_{b I}(C(K))$, which means that its restriction to $n B_{C(K)}$ belongs to $\mathcal{H}_{I}\left(n B_{C(K)}\right)$ for all $n$, (see $[5])$.

The converse of Proposition 1.1 does not hold even for homogeneous polynomials: the condition for an integral polynomial $P$ to be orthogonally additive is that it factor through $R_{k}$, i.e.

$$
\sum_{i} a_{i} x_{i}^{k}=0 \text { on } K \Rightarrow \sum_{i} a_{i} P\left(x_{i}\right)=0 .
$$

Now consider the analogous situation for an integral holomorphic map $f: B_{C(K)}^{\circ} \rightarrow \mathbb{C}$. Take $X$ the closed subspace of $C\left(B_{C(K)^{\prime}}\right)$ spanned by 
$\left\{\frac{\widehat{x}}{1-\widehat{x}}:\|x\|<1\right\}$, and $R: X \rightarrow C(K)$ given by $R\left(\frac{\widehat{x}}{1-\widehat{x}}\right)=\frac{x}{1-x}$. Then $R$ is again well-defined and continuous, and by composition with $R$, all maps of the form

$$
f(x)=\int_{K} \frac{x}{1-x} d \mu
$$

are integral (and $f(0)=0$ ). However, not all orthogonally additive bounded-type holomorphic functions are of this form. To obtain such a representation, one would have to construct a measure over $K$, and here the obstruction is algebraic: the spaces $R_{k}\left(X_{k}\right)$ are not 'independent' in $C(K)$. The following example shows that for non-homogeneous polynomials there is no such representation with any holomorphic $\Phi: \mathbb{C} \rightarrow \mathbb{C}$.

Example 1. Take $K$ to be the closed unit disc in $\mathbb{C}$. There is no entire function $\Phi: \mathbb{C} \rightarrow \mathbb{C}$ such that $h(x)=\Phi \circ x$ factors all degree two orthogonally additive polynomials over $C(K)$.

Proof. Suppose there were such a function $\Phi$, i.e., given an orthogonally additive polynomial $P$, there is a measure $\mu$ such that

$$
P(x)=\int_{K} \Phi \circ x d \mu \quad \text { for } x \in C(K) .
$$

Write the Taylor series of $\Phi$ at 0 :

$$
\Phi(z)=\sum_{k=0}^{\infty} c_{k} z^{k}
$$

and choose $a$ and $b$ in $K$ such that $c_{2} a^{2} \neq c_{1} b^{2}$. Define $P: C(K) \rightarrow \mathbb{C}$ by $P(x)=x(a)+x(b)^{2}$. Clearly, $P$ is orthogonally additive, so let $\mu$ be as above. Now for any $\lambda$,

$$
x(a) \lambda+x(b)^{2} \lambda^{2}=P(\lambda x)=\int_{K} \Phi \circ \lambda x d \mu=\sum_{k=0}^{\infty} c_{k} \int_{K} x^{k} d \mu \lambda^{k}
$$

and thus

$$
x(a)=c_{1} \int_{K} x d \mu, \quad \text { and } \quad x(b)^{2}=c_{2} \int_{K} x^{2} d \mu \text { for } x \in C(K) .
$$

However, if we take $x(z)=\frac{c_{2}}{c_{1}} z^{2}$ and $y(z)=z\left(\right.$ and thus $\left.c_{1} x=c_{2} y^{2}\right)$,

$$
\frac{c_{2}}{c_{1}} a^{2}=x(a)=c_{1} \int_{K} x d \mu=c_{2} \int_{K} y^{2} d \mu=y(b)^{2}=b^{2},
$$

which contradicts our choice of $a$ and $b$. 


\section{Characterization of orthogonally additive HOLOMORPHIC FUNCTIONS OF BOUNDED TYPE}

For any Borel measure $\mu$ on $\mathrm{K}$, we will write $f<<\mu$, if $\mu(A)=0$ implies $f_{A}=0$. Also, we will say that $h: C(K) \rightarrow L^{1}(\mu)$ is a power series function if

$$
h(x)=\sum_{k=1}^{\infty} g_{k} x^{k}
$$

with $g_{k}$ 's in $L^{1}(\mu)$. Note that this is a very special type of holomorphic function, as its definition uses the algebra structure of $C(K)$. We then have the following.

Theorem 2.1. Given $f \in \mathcal{H}_{b}(C(K))$, orthogonally additive, $f<<\mu$ if and only if there exists a power series function $h \in \mathcal{H}_{b}\left(C(K), L^{1}(\mu)\right)$ such that

$$
f(x)=\int_{K} h(x)(t) d \mu(t) \quad \text { for all } x \in C(K) .
$$

Proof. Consider the Taylor series of $f$ about $0: f=\sum_{k} P_{k}$. Note that $\bar{f}=\sum_{k} \overline{P_{k}}$ in $C(K)^{\prime \prime}$. Thus for any Borel set $A$,

$$
f_{A}(x)=\bar{f}\left(1_{A} x\right)=\sum_{k=0}^{\infty} \overline{P_{k}}\left(1_{A} x\right)=\sum_{k=0}^{\infty} P_{k A}(x) .
$$

Since $\left\|P_{k A}\right\| \leq\left\|P_{k}\right\|$ by $\left[3\right.$, Lemma 1.2.] $f_{A} \in \mathcal{H}_{b}(C(K))$

$\Rightarrow)$ Now suppose $f<<\mu$. Since $f_{A}=0$ if and only if $P_{k A}=0$ for all $k$, we have $P_{k}<<\mu$ for all $k$. Also, since $f$ is orthogonally additive, so is $P_{k}$ for all $k$. Therefore, by [2] [9], there is a measure $\mu_{k}$ such that

$$
P_{k}(x)=\int_{K} x^{k} d \mu_{k}
$$

but since $P_{k}<<\mu, \mu_{k}<<\mu$ : indeed, if $\mu(A)=0$,

$$
\mu_{k}(A)=\int_{K} 1_{A} d \mu_{k}=\overline{P_{k}}\left(1_{A}\right)=P_{k A}(1)=0,
$$

(the second equality by [3, Corollary 2.1.]). Now, by the RadonNikodym theorem there is a $g_{k} \in L^{1}(\mu)$ such that

$$
P_{k}(x)=\int_{K} x^{k} g_{k} d \mu \text {. }
$$


We prove that $\left\|g_{k}\right\|_{L^{1}(\mu)} \leq\left\|P_{k}\right\|$ for each $k$ : choose a representative $g_{k}$ and consider the function

$$
t \mapsto\left\{\begin{array}{cl}
\left(\frac{\overline{g_{k}}(t)}{\left|g_{k}(t)\right|}\right)^{1 / k} & , \text { if } g_{k}(t) \neq 0, \\
0 & , \text { if } g_{k}(t)=0,
\end{array}\right.
$$

where, in taking $k$-th root we have chosen any branch. This function, though not continuous, is Borel measurable, and has image in the closed unit disc. It is an element of $C(K)^{\prime \prime}$. Using [3, Corollary 2.1.] again we have

$$
\begin{aligned}
\left\|g_{k}\right\|_{L^{1}(\mu)} & =\int_{K}\left|g_{k}\right| d \mu=\int_{K}\left[\left(\frac{\overline{g_{k}}(t)}{\left|g_{k}(t)\right|}\right)^{1 / k}\right]^{k} g_{k} d \mu \\
& =\overline{P_{k}}\left(\left(\frac{\overline{g_{k}}(t)}{\left|g_{k}(t)\right|}\right)^{1 / k}\right) \leq\left\|\overline{P_{k}}\right\|=\left\|P_{k}\right\|
\end{aligned}
$$

this last equality by [4]. Now define $h: C(K) \rightarrow L^{1}(\mu)$ by

$$
h(x)=\sum_{k=1}^{\infty} g_{k} x^{k} .
$$

This series converges absolutely:

$$
\begin{aligned}
\left\|\sum_{k=0}^{\infty} g_{k} x^{k}\right\|_{L^{1}(\mu)} & \leq \sum_{k=0}^{\infty}\left\|g_{k} x^{k}\right\|_{L^{1}(\mu)} \\
& =\sum_{k=0}^{\infty} \int_{K}\left|g_{k}\left\|\left.x\right|^{k} d \mu \leq \sum_{k=0}^{\infty}\right\| g_{k}\left\|_{L^{1}(\mu)}\right\| x \|_{\infty}^{k}\right. \\
& \leq \sum_{k=0}^{\infty}\left\|P_{k}\right\|\|x\|^{k}<\infty \text { for all } x .
\end{aligned}
$$

Thus $h$ is a power series function and is clearly bounded on bounded subsets of $C(K)$. So $h \in \mathcal{H}_{b}\left(C(K), L^{1}(\mu)\right)$, and

$$
\begin{aligned}
& f(x)=\sum_{k=0}^{\infty} P_{k}(x)=\sum_{k=0}^{\infty} \int_{K} g_{k} x^{k} d \mu=\int_{K} \sum_{k=0}^{\infty} g_{k} x^{k} d \mu=\int_{K} h(x) d \mu . \\
\Leftarrow) \text { If } \mu(A)=0, & f_{A}(x)=\bar{f}\left(1_{A} x\right)=\int_{K} \bar{h}\left(1_{A} x\right) d \mu=\int_{K} h(x) 1_{A} d \mu=\int_{A} h(x) d \mu=0,
\end{aligned}
$$

so $f<<\mu$. The second equality holds because $h$ is a power series function of bounded type. Indeed, $f=\sum_{k} P_{k}=\int_{K} h d \mu$. Since $h$ is a 
power series, $P_{k}(x)=\int_{K} g_{k} x^{k} d \mu$. The proof of [3, Corollary 2.1] then shows that $\overline{P_{k}}\left(1_{A} x\right)=\int_{A} g_{k} x^{k} d \mu$, and the result follows.

Proposition 2.2. If $f$ is orthogonally additive, then for some Borel measure $\mu, f<<\mu$.

Proof. Say $f=\sum_{k} P_{k}$ is the Taylor expansion of $f$ at zero. Since $f$ is orthogonally additive, so are all the $P_{k}$ 's. Thus there are Borel measures $\mu_{k}$ for which

$$
P_{k}(x)=\int_{K} x^{k} d \mu_{k}
$$

Now, $P_{k A}(x)=\overline{P_{k}}\left(1_{A} x\right)=\int_{A} x^{k} d \mu_{k}$, so $P_{k A}(1)=\mu_{k}(A)$ and $\left|\mu_{k}\right| \leq$ $\left\|P_{k}\right\|$. Therefore $\left(\left|\mu_{k}\right|\right)$ is summable, and we can define $\mu=\sum_{k}\left|\mu_{k}\right|$. For any $A, \mu(A)=\sum_{k}\left|\mu_{k}\right|(A)$, so $\mu_{k}<<\mu$ for all $k$. This implies that $P_{k}<<\mu$ for all $k$, and hence $f<<\mu$.

We have the following characterization of orthogonally additive holomorphic functions of bounded type.

Theorem 2.3. $f \in \mathcal{H}_{b}(C(K))$ is orthogonally additive if and only if there is a Borel measure $\mu$ and a power series function $h \in \mathcal{H}_{b}\left(C(K), L^{1}(\mu)\right)$ such that

$$
f(x)=\int_{K} h(x)(t) d \mu(t) \quad \text { for all } x .
$$

Proof. $\Rightarrow$ ) Immediate from the preceding results.

$\Leftarrow)$ Note that all power series functions are orthogonally additive, for if $x$ and $y$ are orthogonal, $(x+y)^{k}=x^{k}+y^{k}$ for all $k \geq 1$, so

$h(x+y)=\sum_{k=1}^{\infty} g_{k}(x+y)^{k}=\sum_{k=1}^{\infty} g_{k}\left(x^{k}+y^{k}\right)=\sum_{k=1}^{\infty} g_{k} x^{k}+\sum_{k=1}^{\infty} g_{k} y^{k}=h(x)+h(y)$.

Now if $f(x)=\int_{K} h(x) d \mu$, orthogonal additivity of $f$ follows from linearity of the integral. Also, if $\|x\| \leq c$,

$$
|f(x)| \leq \sum_{k=1}^{\infty}\left\|g_{k}\right\|_{L^{1}(\mu)} c^{k},
$$

so $f$ is bounded on bounded sets.

\section{REFERENCES}

[1] R. Aron and P. Berner, A Hahn-Banach extension theorem for analytic mappings. Bull. Soc. Math. France, 106 (1) (1978), 3-24.

[2] Y. Benyamini, S. Lassalle and J. G. Llavona, Homogeneous orthogonallyadditive polynomials on Banach lattices. Bull. Lond. Math. Soc., 38 (3) (2006), 459-469. 
[3] D. Carando, S. Lassalle and I. Zalduendo, Orthogonally additive polynomials over $C(K)$ are measures - a short proof. Integral Eq. and Operator Theory, 56 (2006), 597-602.

[4] A. Davie and T. Gamelin, A theorem on polynomial-star approximation. Proc. Amer. Math. Soc. 106 (2) (1989), 351-356.

[5] V. Dimant, P. Galindo, M. Maestre and I. Zalduendo, Integral holomorphic functions. Studia Math. 160 (2004), 83-99.

[6] S. Dineen, Holomorphic functions on a Banach space. Bull. Amer. Math. Soc. 76 (1970), 883-886.

[7] S. Dineen, Holomorphy types on a Banach space. Studia Math. 39 (1971), 241-288.

[8] S. Dineen, Complex Analysis on Infinite Dimensional Spaces. Springer-Verlag, 1999.

[9] D. Pérez-García and I. Villanueva, Orthogonally additive polynomials on spaces of continuous functions. J. Math. Anal. Appl. 306 (1) (2005), 97-105.

Depto. de Matemática, Pab. I, Fac. de Cs. Exactas y Naturales, Universidad de Buenos Aires, (1428) Buenos Aires, Argentina

E-mail address: dcarando@dm.uba.ar

Depto de Matemática, Pab. I, Fac. de Cs. Exactas y Naturales, Universidad de Buenos Aires, (1428) Buenos Aires, Argentina

E-mail address: slassall@dm.uba.ar

Depto. de Matemática, Universidad Torcuato Di Tella, Miñones 2177 (C1428ATG), Buenos Aires, Argentina

E-mail address: nacho@utdt.edu 